\title{
On Products Of Various Graphs And Their Spectra: A Survey
}

\author{
Ramakrishnan K. O. ${ }^{1}$, Santhosh G. O. ${ }^{2}$, Rajeevan P. ${ }^{3}$, \\ ${ }^{1}$ Assistant Professor, Department of Mathematics, K.M.M Govt. Women's College, Kannur. \\ ${ }^{2,3}$ Assistant Professor, Department of Mathematics, Govt. Brennen College, Thalassery-6 \\ Email: ramkomaths@gmail.com ${ }^{1}$,go.santhosh.go@gmail.com ${ }^{2}$,rajeevtpba@gmail.com ${ }^{3}$
}

\begin{abstract}
In this survey, we highlight and enlist vivid results related mainly to the product of three graph structures, namely graphs, signed graphs and gain graphs and garner facts relating to the spectra of their adjacency and Laplacian matrices. Cartesian product, lexicographic product, NEPS (Non-extendable p sums) are some of the products which come under the main discussion. Scope for further extending the results to the realm of skew-gain graphs, which encompass and generalize the above structures, comes as concluding remarks.
\end{abstract}

Keywords- Graph, Signed graph, Gain Graph, Laplacian matrix, Graph spectra. Mathematics Subject Classification (2010): 05C22, 05C50, 05 C76.

\section{INTRODUCTION}

We survey various graph products and explore the results relating to their spectra with respect to the corresponding to the adjacency and Laplacian (or Kirchhoff)matrices. All graphs in this article are simple and finite. We assume that the readers are aware of basic results in graph theory, the details of which can be had from [19] or any other well known resources. Signed graphs (also called sigraphs), with positive or negative labels on the edges, are much studied in the literature because of their use in modeling a variety of physical and sociopsychological processes (see [3] and [1, 14]) and also because of their interesting connections with many classical mathematical systems (see [29]). Formally, a signed graph is an ordered pair $\Sigma=(\mathrm{G}, \sigma)$ where $\mathrm{G}=$ $(\mathrm{V}, \mathrm{E})$ is a graph called the underlying graph of $\Sigma$ and $\sigma: \mathrm{E} \rightarrow\{+1,-1\}$, called a signing (also called a signature), is a function from the edge set $\mathrm{E}$ of $\mathrm{G}$ into the set $\{+1,-1\}$ of signs. The sign of a cycle in a signed graph is the product of the signs of its edges. Thus a cycle is positive if and only if it contains even number of negative edges. A signed graph $\Sigma$ is said to be balanced (or cycle balanced) if all of its cycles are positive.

A signed graph is all-positive (respectively, allnegative) if all of its edges are positive (negative); further, it is said to be homogeneous if it is either allpositive or all negative. A graph can be considered to be a homogeneous signed graph; thus signed graphs becomea generalization of graphs. The notion of signed graphs is further generalized to gain graphs as follows. A gain graph is a graph where the edges are given some orientation and labelled with the elements (called gains) from a group so that the gains are inverted when we reverse the direction of the edges. Throughout this paper, $\mathrm{F} \times$, where $\mathrm{F}$ is a field of characteristic zero, denotes the multiplicative group of non-zero elements in $F$. The notation $\Phi=(\mathrm{G}, \mathrm{F} \times, \phi)$ denotes a gain graph $\Phi$ with underlying graph $\mathrm{G}$, underlying group $\mathrm{F} \times$ and the gain function $\phi$. For definition and other details for gain graphs, one may refer to [29].

The Cartesian product $\Sigma_{1} \times \Sigma_{2}$ of two signed graphs $\Sigma_{1}$ $=\left(\mathrm{V}_{1}, \mathrm{E}_{1}, \sigma_{1}\right)$ and $\Sigma_{2}=\left(\mathrm{V}_{2}, \mathrm{E}_{2}, \sigma_{2}\right)$ is a generalization of the Cartesian product of ordinary graphs (see $[6$, Section 2.5]). It is defined as the signed graph $\left(\mathrm{V}_{1} \times\right.$ $\mathrm{V}_{2}, \mathrm{E}, \sigma$ ) where the edge set $\mathrm{E}$ is that of the Cartesian product of underlying unsigned graphs and the signature function $\sigma$ for the labeling of the edges is defined by

$$
\sigma\left(\left(u_{i}, v_{j}\right),\left(u_{k}, v_{l}\right)\right)=\left\{\begin{array}{l}
\sigma_{1}\left(u_{i}, u_{k}\right) \text { if } j=l, \\
\sigma_{2}\left(v_{j}, v_{l}\right) \text { if } i=k .
\end{array}\right.
$$

The adjacency matrix and the Laplacian matrix of a signed graph are immediate generalizations of familiar matrices from ordinary, unsigned graph theory ([6]). Thus, if $\Sigma=(\mathrm{G}, \sigma)$ is a signed graph where $\mathrm{G}=(\mathrm{V}, \mathrm{E})$ with $\mathrm{V}=\left\{\mathrm{v}_{1}, \mathrm{v}_{2}, \ldots, \mathrm{v}_{\mathrm{n}}\right\}$, its adjacency matrix $\mathrm{A}(\Sigma)=$ $\left(a_{i j}\right)_{n \times n}$ is defined as

$$
a_{i j}=\left\{\begin{array}{c}
\sigma\left(u_{i}, v_{j}\right), \text { if } u_{i} \wedge v_{j} \text { areadjacent } \\
0, \text { otherwise }
\end{array}\right.
$$

The Laplacian matrix (or Kirchhoff matrix or admittance matrix) of a signed graph $\Sigma$, denoted by $\mathrm{L}(\Sigma)($ or $\mathrm{K}(\Sigma))$, is $\mathrm{D}(\Sigma)-\mathrm{A}(\Sigma)$ where $\mathrm{D}(\Sigma)$ is the diagonal matrix of the degrees of vertices of $\Sigma$. The ordinary adjacency and Laplacian matrices of a graph $\mathrm{G}$ are identical with those of the all-positive signed graph $+\mathrm{G}$. The so-called signless Laplacian of $\mathrm{G}$ ([7]) is the Laplacian matrix of the all-negative graph $-\mathrm{G}$. Eigenvalues of the adjacency matrix, the Laplacian matrix and the signless Laplacian matrix of a graph have been widely used to characterize properties of a graph and extract some useful information from its structure. The eigenvalues of the adjacency matrix of a graph are often referred to as the eigenvalues of the graph and those of the Laplacian matrix as the Laplacian eigenvalues. file including postscript files for figures. 


\section{Available online at www.ijrat.org}

\section{NOTION OF BALANCE AND BALANCED COMPONENTS}

For a signed graph $\Sigma$, the quantity $\mathrm{c}(\Sigma)=\mathrm{c}(\mathrm{G})$ is the number of connected components of the underlying graph and $c_{b}(G)$ is the number of its components that are bipartite. The quantity $b(\Sigma)$ is the number of connected components of $\Sigma$ that are balanced. An essential lemma in signed graph theory is a characterization of balance by switching, which when expressed in terms of the adjacency matrix takes the following form:

Lemma 2.1 ([27]). $\Sigma$ is balanced if and only if there is a diagonal matrix $S$ with diagonal elements \pm 1 such that $\mathrm{SA}(\Sigma) \mathrm{S}$ is non-negative. Then $\mathrm{SA}(\Sigma) \mathrm{S}=\mathrm{A}(\mathrm{G})$ where $\mathrm{G}$ is the underlying graph of $\Sigma$

The negation of a signed graph $\Sigma=(\mathrm{G}, \sigma)$, denoted by $-\Sigma=(\mathrm{G},-\sigma)$, is the same graph with all signs reversed. The adjacency matrices are related by $\mathrm{A}(-\Sigma)=-\mathrm{A}(\Sigma)$. The adjacency matrix $A(\Phi)=\left(a_{i j}\right)$ of a gain graph $\Phi=\left(\mathrm{G}, \mathrm{F}^{\times}, \phi\right)$, is defined as

$$
a_{i j}= \begin{cases}\varphi\left(u_{i}, v_{j}\right), & \text { if } \overrightarrow{u_{1} v_{j}} \in \vec{E} \\ 0, & \text { otherwise }\end{cases}
$$

and $\mathrm{a}_{\mathrm{ji}}=\varphi\left(\mathrm{v}_{\mathrm{i}}, \mathrm{v}_{\mathrm{j}}\right)^{-1}$ where $0_{\mathrm{F}} \quad$ is the additive identity in the field. If there is no scope for confusion we drop the subscript $\mathrm{F}$ and write the additive identity in $\mathrm{F}$ simply as 0 and the multiplicative identity of $\mathrm{F}$ as 1 . Also, whenever we deal with the matrix representation of a gain graph, we take the underlying group to be $\mathrm{F}$ $x$ and on all other occasions the same to be a general group $\Gamma$, or an abelian group, if the situation demands so. The characteristic polynomial of $\Phi$, denoted by $\Psi(\Phi, \mathrm{x})$ or simply as $\Psi(\Phi)$, is defined as the characteristic polynomial $\operatorname{det}(\mathrm{xI}-\mathrm{A}(\Phi)) \in \mathrm{F}[\mathrm{x}]$ of $\mathrm{A}(\Phi)$, where I is the identity matrix of order as that of $\mathrm{A}(\Phi)$. The zeros of $\Psi(\Phi, \mathrm{x})$, in $\mathrm{F}$ or in its splitting field, are called eigenvalues of $\Phi$. For more details on matrices with entries from a field, their determinants, eigenvalues and eigenvectors, the reader may refer to [22].

A signed graph can be taken as a gain graph with the underlying group as a gain graph over the multiplicative subgroup $\{1,-1\}$ of any field F. A graph can be regarded as a gain graph with the underlying group as $\{1\}$. The gain $\varphi(\mathrm{C})$, of a cycle $\mathrm{C}$ : $\mathrm{v}_{0} \mathrm{v}_{1} \ldots \mathrm{v}_{\mathrm{n}} \mathrm{v}_{0}$, is the product $\varphi\left(\mathrm{v}_{0} \mathrm{v}_{1}\right) \varphi\left(\mathrm{v}_{1} \mathrm{v}_{2}\right) \ldots \varphi\left(\mathrm{v}_{\mathrm{n}} \mathrm{v}_{0}\right)$ of gains of its edges. The balance notion defined above in the case of signed graphs are extended naturally to gain graphs. Recall that the spectrum of a graph or signed graph is the spectrum of its adjacency matrix and that the spectrum is the list of eigenvalues with their multiplicities. The Laplacian spectrum is the spectrum of the Laplacian matrix. Acharya's theorem, following, gives a spectral criterion for balance in signed graphs.
Theorem 2.2 ([14]). If $\Sigma=(\mathrm{G}, \sigma)$ is a signed graph, then $\Sigma$ is balanced if and only if $G$ and $\Sigma$ have the same spectrum.

The following theorem, found in [11], is a significant extension of the above theorem for signed graphs to gain graphs.

Theorem 2.3 ([11]). If $\Phi=\left(\mathrm{G}, \mathrm{F}^{\times}, \varphi\right)$ is a gain graph where $G=(V, E)$, then $\Phi$ is balanced if, and only if, $\Psi(\Phi, \mathrm{x})=\Psi(\mathrm{G}, \mathrm{x})$.

2.1 Cartesian Product of two gain graphs

Now we enlist results for the cartesian product of two gain graphs and a characterization theorem for balance when the group for assigning the edge gain is abelian. Given two gain graphs $\Phi_{1}=\left(\mathrm{V}_{1}, \mathrm{E}_{1}, \Gamma, \varphi_{1}\right)$ and $\Phi_{2}=\left(\mathrm{V}_{2}, \mathrm{E}_{2}, \Gamma, \varphi_{2}\right)$, their cartesian product $\Phi_{1} \times \Phi_{2}$ is defined as the gain graph $\left(\mathrm{V}_{1} \times \mathrm{V}_{2}, \mathrm{E}, \Gamma, \varphi\right)$ where the edge set $\mathrm{E}$ is that of the cartesian product of underlying graphs and the gain function $\varphi$ for the labeling of the edges is defined by

$$
\sigma\left(\left(u_{i}, v_{j}\right),\left(u_{k}, v_{l}\right)\right)=\left\{\begin{array}{l}
\varphi_{1}\left(u_{i}, u_{k}\right) \text { if } v_{j}=v_{l} \wedge u_{i} u_{k} \in E \\
\varphi_{2}\left(v_{j}, v_{l}\right) \text { if } u_{i}=u_{k} \wedge v_{j} v_{l} \in E
\end{array}\right.
$$

Now we need an important notion called switching of gain graphs (For more details see [29]). If $\zeta: \mathrm{V} \rightarrow \Gamma$ is a function called switching function, then switching of the gain graph $\Phi=(G, \Gamma, \varphi)$ by $\zeta$ means changing $\varphi$ to $\varphi \zeta$ defined by:

$$
\varphi^{\zeta}(\mathrm{uv})=\zeta(\mathrm{u}) \varphi(\mathrm{uv})(\zeta(\mathrm{v}))^{-1} .
$$

The switched graph, denoted by $\Phi^{\zeta}$, is the gain graph $\Phi^{\zeta}=\left(\mathrm{G}, \Gamma, \varphi^{\zeta}\right)$. We call two gain graphs $\Phi_{1}=\left(\mathrm{G}, \Gamma, \varphi_{1}\right)$ and $\Phi_{2}=\left(G, \Gamma, \varphi_{2}\right)$ to be switching equivalent, if there exists a switching function $\zeta: \mathrm{V} \rightarrow \Gamma$ such that $\Phi_{1}=\Phi_{2}$. It can be seen that switching preserves many features of the two gain graphs including their eigenvalues[29]. Indeed, the following is a very important result.

Lemma 2.4 ([27]). A gain graph is balanced if, and only if, it can be switched to an all-identity gain graph.

Theorem 2.5. ([9]) If $\Phi_{1}=\left(\mathrm{V}_{1}, \mathrm{E}_{1}, \Gamma, \varphi_{1}\right)$ and $\Phi_{2}=\left(\mathrm{V}_{2}, \mathrm{E}_{2}, \Gamma, \varphi_{2}\right)$ are two gain graphs where $\Gamma$ is abelian, then their cartesian product $\Phi_{1} \times \Phi_{2}$, is balanced if, and only if, $\Phi_{1}$ and $\Phi_{2}$ are both balanced.

The condition that $\Gamma$ must be abelian cannot be relaxed, since in the case of the two gain paths $\Phi_{\mathrm{P} 2}=$ $\left(\mathrm{P}_{2}, \Gamma, \varphi_{1}\right)$ and $\Phi_{\mathrm{P} 2}^{\prime}=\left(\mathrm{P}_{2}, \Gamma, \varphi_{2}\right)$ where $\Gamma$ is any nonabelian group and if $\alpha$ and $\beta$ are any two noncommuting elements of $\Gamma$ such that $\varphi_{1}\left(\mathrm{u}_{1} \mathrm{u}_{2}\right)=\alpha$ and $\varphi_{2}\left(\mathrm{v}_{1} \mathrm{v}_{2}\right)=\beta$, then $\Phi_{\mathrm{P} 2} \times \Phi_{\mathrm{P} 2}^{\prime}$ is unbalanced even though the constituent gain paths are balanced.

Balance in the case of the cartesian product of several gain graphs can be discussed in a similar way, which we do in Section 3.3. 


\section{Available online at www.ijrat.org}

\subsection{Lexicographic Product Of Two Gain Graphs}

If $\mathrm{G}_{1}=\left(\mathrm{V}_{1}, \mathrm{E}_{1}\right)$ and $\mathrm{G}_{2}=\left(\mathrm{V}_{2}, \mathrm{E}_{2}\right)$ are two graphs, their lexicographic product $\mathrm{G}_{1}\left[\mathrm{G}_{2}\right]$ is defined as the graph with the vertex set $\mathrm{V}_{1} \times \mathrm{V}_{2}$ and the vertices $\mathrm{u}=\left(\mathrm{u}_{\mathrm{i}}, \mathrm{v}_{\mathrm{j}}\right)$ and $\mathrm{v}=\left(\mathrm{u}_{\mathrm{k}}, \mathrm{v}_{\mathrm{l}}\right)$ are adjacent whenever $\mathrm{u}_{\mathrm{i}}$ is adjacent to $\mathrm{u}_{\mathrm{k}}$ or when $\mathrm{u}_{\mathrm{i}}=\mathrm{u}_{\mathrm{k}}$ and $\mathrm{v}_{\mathrm{j}}$ is adjacent to $\mathrm{v}_{\mathrm{l}}$. We shall extend this definition to gain graphs. We define the lexicographic product $\Phi_{1}\left[\Phi_{2}\right]$ (also called composition) of two gain graphs $\Phi_{1}=\left(\mathrm{V}_{1}, \mathrm{E}_{1}, \Gamma, \phi_{1}\right)$ and $\Phi_{2}=\left(\mathrm{V}_{2}, \mathrm{E}_{2}, \Gamma, \varphi_{2}\right)$ as the gain graph $\left(\mathrm{V}_{1} \times \mathrm{V}_{2}, \mathrm{E}, \Gamma, \varphi\right)$ where the edge set $\mathrm{E}$ is that of the lexicographic product of underlying graphs and the edge gain assigning function $\phi$ is defined byIf $\mathrm{G}_{1}=\left(\mathrm{V}_{1}, \mathrm{E}_{1}\right)$ and $\mathrm{G}_{2}=\left(\mathrm{V}_{2}, \mathrm{E}_{2}\right)$ are two graphs, their lexicographic product $\mathrm{G}_{1}\left[\mathrm{G}_{2}\right]$ is defined as the graph with the vertex set $\mathrm{V}_{1} \times \mathrm{V}_{2}$ and the vertices $\mathrm{u}=\left(\mathrm{u}_{\mathrm{i}}, \mathrm{v}_{\mathrm{j}}\right)$ and $\mathrm{v}=\left(\mathrm{u}_{\mathrm{k}}, \mathrm{v}_{\mathrm{l}}\right)$ are adjacent whenever $u_{i}$ is adjacent to $u_{k}$ or when $u_{i}=u_{k}$ and $v_{j}$ is adjacent to $v_{1}$. We shall extend this definition to gain graphs. We define the lexicographic product $\Phi_{1}\left[\Phi_{2}\right]$ (also called composition) of two gain graphs $\Phi_{1}=\left(\mathrm{V}_{1}, \mathrm{E}_{1}, \Gamma, \varphi_{1}\right)$ and $\Phi_{2}=\left(\mathrm{V}_{2}, \mathrm{E}_{2}, \Gamma, \varphi_{2}\right)$ as the gain graph $\left(\mathrm{V}_{1} \times \mathrm{V}_{2}, \mathrm{E}, \Gamma, \varphi\right)$ where the edge set $\mathrm{E}$ is that of the lexicographic product of underlying graphs and the edge gain assigning function $\varphi$ is defined by

$$
\sigma\left(\left(u_{i}, v_{j}\right),\left(u_{k}, v_{l}\right)\right)=\left\{\begin{array}{c}
\varphi_{1}\left(u_{i}, u_{k}\right) \text { if } u_{i} \neq u_{k} \wedge u u_{k} \in E \\
\varphi_{2}\left(v_{j}, v_{l}\right) \text { if } u_{i}=u_{k} \wedge v_{j} v_{l} \in E
\end{array}\right.
$$

Theorem 2.6.([11]) If $\Phi_{1}$ and $\Phi_{2}$ are two gain graphs over the same abelian group $\Gamma$, then their lexicographic product or composition $\Phi_{1}\left[\Phi_{2}\right]$ is balanced if, and only if, $\Phi_{1}$ is balanced and $\Phi_{2}$ is an all-identity gain graph.

\section{SPECTRA OF PRODUCT OF GRAPHS}

In this section we deal with spectra of product of graphs for which we require some introductory remarks. The incidence matrix of a signed graph $\Sigma$ with $n$ vertices and $m$ edges ([27]) is the $n \times m$ matrix $\mathrm{H}(\Sigma)=\left[\eta_{i j}\right]$ in which $\eta_{\mathrm{ik}}=0$ if $\mathrm{v}_{\mathrm{i}}$ is not incident with $e_{k}$, and $\eta_{i k}= \pm 1$ if $v_{i}$ is incident with $e_{k}$, and such that for an edge $\mathrm{v}_{\mathrm{i}} \mathrm{v}_{\mathrm{j}}$, the product $\eta_{\mathrm{ik}} \eta_{\mathrm{jk}}=-\sigma\left(\mathrm{v}_{\mathrm{i}} \mathrm{v}_{\mathrm{j}}\right)$. The incidence matrix is uniquely determined only up to multiplication of columns by -1 , but that ambiguity does not affect any of the properties of interest to us. In particular, the incidence matrix always satisfies the Kirchhoff equation $\mathrm{H}(\Sigma) \mathrm{H}(\Sigma)^{\mathrm{T}}=\mathrm{L}(\Sigma)$. For that reason the Laplacian matrix is positive semi-definite.

Lemma 3.1 ([9]). The incidence matrix and the Laplacian matrix of a signed graph $\Sigma$ both have rank $n$ $-b(\Sigma)$.

For a graph $\mathrm{G}$, the Laplacian $\mathrm{L}(\mathrm{G})=\mathrm{L}(+\mathrm{G})$ has rank $\mathrm{n}-\mathrm{c}(\mathrm{G})$ and the signless Laplacian $\mathrm{Q}(\mathrm{G})=\mathrm{L}(-\mathrm{G})$ has rank $n-c_{b}(G)$.

We take note of the special case in which the underlying graph $\mathrm{G}$ is regular. The following lemma generalizes the well known fact that, for a k-regular graph, the smallest eigenvalue is $-\mathrm{k}$ occurring with multiplicity $c_{b}(G)$, the largest eigenvalue is $k$ with multiplicity $c(G)$ and the other eigenvalues fall into the open interval $(-\mathrm{k}, \mathrm{k})$.

Lemma 3.2. ([9])Assume $\Sigma$ has underlying graph $G$ which is regular of degree k. Let the eigenvalues of $\Sigma$ be $\lambda_{1}, \lambda_{2}, \ldots, \lambda_{n}$ in weakly increasing order. Then $\lambda 1, \ldots, \lambda \mathrm{b}(-\Sigma)=-\mathrm{k},-\mathrm{k}<\lambda \mathrm{b}(-\Sigma)+1, \ldots, \lambda_{\mathrm{n}}-\mathrm{b}(\Sigma)<\mathrm{k}$ and $\lambda \mathrm{n}-\mathrm{b}(\Sigma)+1, \ldots, \lambda_{\mathrm{n}}=\mathrm{k}$.

The Laplacian eigenvalues of $\Sigma$ are $\lambda_{i}^{\mathrm{L}}=\mathrm{k}-\lambda_{\mathrm{i}}$, including $2 \mathrm{k}$ with multiplicity and 0 with multiplicity $b(\Sigma)$. The Laplacian energy equals the energy.

Lemma 3.2 raises the question of whether it is possible to have $b(-\Sigma)>n-b(\Sigma)$, since if that is the case and k $6=0$, then there is a contradiction in the notation of the lemma. By Lemma 3.4, a contradiction of that kind in Lemma 3.2 is not a problem because $\mathrm{b}(-\Sigma)>\mathrm{n}-\mathrm{b}(\Sigma)$ implies $\mathrm{k}=0$ and then all eigenvalues are $0=\mathrm{k}=-\mathrm{k}$.

Lemma 3.3. ([9]) Both $\Sigma$ and $-\Sigma$ are balanced if and only if the underlying graph $\mathrm{G}$ is bipartite and $\Sigma$ or $-\Sigma$ is balanced.

Lemma 3.4.([9]) $\mathrm{b}(-\Sigma)+\mathrm{b}(\Sigma) \leq \mathrm{n}$ except possibly when the number of isolated vertices is greater than the number of components with order at least 3 .

In particular, if the underlying graph $\mathrm{G}$ is k-regular and $\mathrm{b}(-\Sigma)+\mathrm{b}(\Sigma)>\mathrm{n}$ then $\mathrm{k}=0$.

\subsection{Kronecker Product Of Matrices And Their Applications}

The identity matrix of order $n$ is denoted by $\mathrm{I}_{\mathrm{n}}$. The Kronecker product of matrices $\mathrm{A}=\left[\mathrm{a}_{\mathrm{ij}}\right]_{\mathrm{m} \times \mathrm{p}}$ and $\mathrm{B}$ of orders $\mathrm{m} \times \mathrm{p}$ and $\mathrm{n} \times \mathrm{q}$, respectively, is the matrix $\mathrm{A} \otimes \mathrm{B}$ of order $\mathrm{mn} \times \mathrm{pq}$ defined by

$$
A \otimes B=\left[\begin{array}{cccc}
a_{11} B & a_{12} B & \ldots & a_{1 n} B \\
. . & \ldots & \ldots & \ldots
\end{array}\right]
$$

The Kronecker product is a componentwise operation, that is, $(A \otimes B)\left(A^{\prime} \otimes B^{\prime}\right)=\left(A A^{\prime}\right) \otimes\left(B^{\prime}\right)$. It is also an associative operation; therefore a multiple product $A_{1} \otimes A_{2} \otimes \cdots \otimes A_{v}$ is well defined. Let $A_{i}$ have order $m_{\mathrm{i}} \times n_{\mathrm{i}}$ and elements $\mathrm{a}_{\mathrm{i}, \mathrm{j} \mathrm{k}}$. An element of such a product is indexed by a pair of $v$-tuples, a row index $\mathrm{j}=\left(\mathrm{j}_{1}, \mathrm{j}_{2}, \ldots, \mathrm{j}_{\mathrm{v}}\right)$ and a column index $\mathrm{k}=\left(\mathrm{k}_{1}, \mathrm{k}_{2}, \ldots, \mathrm{k}_{\mathrm{v}}\right)$, where $1 \leq \mathrm{j}_{\mathrm{i}} \leq \mathrm{m}_{\mathrm{i}}$ and $1 \leq \mathrm{k}_{\mathrm{i}} \leq \mathrm{n}_{\mathrm{i}}$. The element $\mathrm{a}_{\mathrm{jk}}$ of the product matrix is

Lemma 3.5 ([6]). Let A and B be square matrices of orders $\mathrm{m}$ and $\mathrm{n}$, respectively, with eigenvalues $\lambda_{\mathrm{i}}$ $(1 \leq \mathrm{i} \leq \mathrm{m})$ and $\mu_{\mathrm{j}}(1 \leq \mathrm{i} \leq \mathrm{n})$. Then the mn eigenvalues of $A \otimes B$ are $\lambda_{i} \mu_{j}$, and those of $A \otimes I_{n}+I_{m} \otimes B$ are $\lambda_{i}$ $+\mu_{\mathrm{j}}$.

The second part of the lemma is due to the fact that $A \otimes I_{n}$ and $I_{m} \otimes B$ are simultaneously diagonalizable. 


\section{Available online at www.ijrat.org}

The first part has an obvious extension to multiple products. That is the first part of the next lemma. The second part is the extension to multiple sums and products.

Lemma 3.6.([6]) Let $A_{i}$, for each $i=1, \ldots, v$, be a square matrix of order $n_{i}$ and let $\lambda_{i j}$ for $1 \leq j \leq n_{i}$ be its eigenvalues. Let $\mathrm{k}_{1}, \ldots, \mathrm{k}_{\mathrm{v}}$ be non-negative integers. Then the $\mathrm{n}_{1} \cdots \mathrm{n}_{v}$ eigenvalues of the Kronecker product $A_{1}^{k_{1}} \otimes \ldots \otimes A_{v}^{k_{v}} \quad$ are $\quad \lambda_{j_{1} \ldots j_{v}}=\lambda_{1 j_{1}}^{k_{1}} \ldots . \lambda_{v j_{v}}^{k_{v}} \quad$ for $1 \leq \mathrm{j}_{\mathrm{i}} \leq \mathrm{n}_{\mathrm{i}}$.

Let $\mathrm{k}_{\mathrm{p}}=\left(\mathrm{k}_{\mathrm{p} 1}, \ldots, \mathrm{k}_{\mathrm{pv}}\right)$ for $1 \leq \mathrm{p} \leq \mathrm{q}$ be vectors of nonnegative integers. Then the $\mathrm{n}_{1} \cdots \mathrm{n}_{v}$ eigenvalues of $\sum_{p=1}^{q} A_{1}^{k_{p 1}} \otimes \ldots \ldots \otimes A_{v}^{k_{p v}}$ are

$$
\lambda_{j 1 \ldots . . j v}=\sum_{p=1}^{q} \lambda_{1 j_{1}}^{k_{p 1}} \ldots \lambda_{v j_{v}}^{k_{p v}} \text { for } 1 \leq \mathrm{j}_{\mathrm{i}} \leq \mathrm{n}_{\mathrm{i}}
$$

Proof. The first part is obvious from Lemma 3.5. The second part is true because the summed matrices commute, so they are simultaneously diagonalizable, they have the same eigenvectors and therefore their eigenvalues can be summed.

\subsection{General Products Of Various Graphs}

Now we define a general product of signed graphs following the idea of Cvetkovi' $c^{\prime}$ for unsigned graphs ([5]) as described in [6, Section 2.5]. We work with signed graphs $\Sigma_{i}=\left(V_{i}, E_{i}, \sigma_{i}\right)$, for $i=1, \ldots, v$, of order $n_{i}$, with underlying graph $\mathrm{G}_{\mathrm{i}}=\left(\mathrm{V}_{\mathrm{i}}, \mathrm{E}_{\mathrm{i}}\right)$, vertex set $\mathrm{V}_{\mathrm{i}}=\left\{\mathrm{v}_{\mathrm{i} 1}, \mathrm{v}_{\mathrm{i} 2}, \ldots, \mathrm{v}_{\mathrm{ini}}\right\}$ and adjacency matrix $\mathrm{A}_{\mathrm{i}}$. We denote the eigenvalues of $\Sigma_{\mathrm{i}}$ by $\lambda_{\mathrm{i} 1}, \lambda_{\mathrm{i} 2}, \ldots, \lambda_{\text {ini }}$. The Laplacian eigenvalues are denoted by a superscript L, as $\lambda_{i j}^{L}$.

The general product is known as the non-complete extended p-sum or NEPS, but we shall call it simply the Cvetkovi'c' product. This product is defined in terms of a set $\mathrm{B}$ of $0 / 1$ vectors, called the basis for the product, such that for every $i \in\{1,2, \ldots, v\}$ there exists at least one $\beta \in \mathrm{B}$ for which $\beta_{\mathrm{i}}=1$; we say $\mathrm{B}$ has support $\{1,2, \ldots, v\}$. First we define a product for one arbitrary vector $\beta=\left(\beta_{1}, \beta_{2}, \ldots, \beta_{v}\right) \in\{0,1\}^{v} \subset Z^{v}$. This product, written $\operatorname{NEPS}\left(\Sigma_{1}, \ldots, \Sigma_{v} ; \beta\right)$, is the signed graph $(\mathrm{V}, \mathrm{E}, \sigma)$ with vertex set

edge set

$$
\mathrm{V}=\mathrm{V} 1 \times \mathrm{V} 2 \times \ldots \ldots \times \mathrm{Vv}
$$

$$
\mathrm{E}=\left\{\left(\mathrm{u}_{1}, \ldots, \mathrm{u}_{\mathrm{v}}\right)\left(\mathrm{v}_{1}, \ldots, \mathrm{v}_{\mathrm{v}}\right): \mathrm{u}_{\mathrm{i}}=\mathrm{v}_{\mathrm{i}} \text { if } \beta_{\mathrm{i}}=0\right. \text { and }
$$$$
\left.\mathrm{u}_{\mathrm{i}} \mathrm{v}_{\mathrm{i}} \in \mathrm{E}_{\mathrm{i}} \text { if } \beta_{\mathrm{i}}=1\right\},
$$

and signature

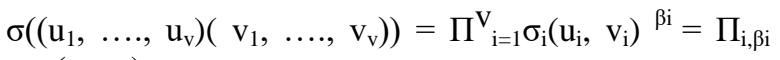
${ }_{=1} \sigma_{\mathrm{i}}\left(\mathrm{u}_{\mathrm{i}}, \mathrm{v}_{\mathrm{i}}\right)$

In the general defnition we have a set

$$
\mathrm{B}=\left\{\beta_{1}, \ldots, \beta_{\mathrm{q}}\right\} \subseteq\{0 ; 1\}^{\mathrm{v}} \backslash\{(0,0, \ldots, 0\}
$$

and we define

$$
\operatorname{NEPS}\left(\sum_{1}, \ldots, \sum_{\mathrm{v}} ; \mathrm{B}\right)=\mathrm{U}_{\beta \in B} \operatorname{NPES}\left(\sum_{1}, \ldots, \sum_{\mathrm{v}} ; \beta\right) .
$$

The underlying graph of $\operatorname{NEPS}\left(\Sigma_{1}, \ldots, \Sigma_{v} ; \mathrm{B}\right)$ is the Cvetkovi'c product $\operatorname{NEPS}\left(\mathrm{G}_{1}, \mathrm{G}_{2}, \ldots, \mathrm{G}_{v} ; \mathrm{B}\right)$ of the underlying graphs as defined in [6, Section 2.5].

In particular, the Cartesian product $\Sigma_{1} \times \Sigma_{2} \times \cdots \times \Sigma_{v}$ arises by taking $\mathrm{B}$ to be the set of all vectors with exactly one coordinate equal to 1 . Another important product, called the strong product or categorical product, is obtained by taking $\mathrm{B}=\{(1,1, \ldots, 1)\}$. $\mathrm{A}$ generalization of both, which could be called the symmetric p-sum (but we think of it as a product), is obtained by taking the set $\mathrm{B}_{\mathrm{p}}$, for $1 \leq \mathrm{p} \leq \mathrm{q}$, which consists of all vectors $\beta$ with exactly $p$ coordinates equal to 1 . An incomplete p-product, where $B \subseteq B_{p}$, has the nice property that

$$
\begin{aligned}
N E P S & \left(\left(-\Sigma_{1}, \ldots,-\Sigma_{v} ; B\right)\right. \\
& =(-1)^{p} \operatorname{NEPS}\left(\left(-\Sigma_{1}, \ldots, \Sigma_{v} ; B\right)\right.
\end{aligned}
$$

For instance in the Cartesian product $\left(-\Sigma_{1}\right) \times \cdots \times\left(-\Sigma_{v}\right)=-\left(\Sigma_{1} \times \cdots \times \Sigma_{v}\right)$.

A final property shows that a Cvetkovi'c' product of all-positive signed graphs is essentially equivalent to the same product of the underlying graphs. Clearly, $N E P S\left(+G_{1}, \ldots,+G_{v} ; B\right)=+N E P S\left(G_{1}, \ldots, G_{v} ; B\right)$

We now provide expressions for the adjacency, degree and Laplacian matrices of the Cvetkovi'c' product of signed graphs in terms of the Kronecker products of the corresponding matrices of the constituent graphs. An important application is the characterization of balance of the product graph.

First we give the way of dealing with the adjacency matrix of the Cvetkovi'c' product, which implies expressions for the eigenvalues. For the eigenvalues of $\Sigma_{\mathrm{i}}$ we write $\lambda_{\mathrm{ij}}, 1 \leq \mathrm{j} \leq \mathrm{n}_{\mathrm{i}}$. This theorem generalizes [6, Theorems 2.21 and 2.23] to signed graphs.

Theorem 3.7. ([9]) Let $\Sigma=\operatorname{NEPS}\left(\Sigma_{1}, \ldots, \Sigma_{v} ; \mathrm{B}\right)$. The adjacency matrix is given by $A(\Sigma)=\sum_{\beta \in \mathrm{B}} \mathrm{A}_{1}^{\beta_{1}} \otimes \ldots \otimes \mathrm{A}_{\mathrm{v}}^{\beta_{\mathrm{v}}}$. The eigen values are $\lambda_{j_{1}-j_{v}}=\sum_{\beta \in B} \lambda_{1_{j 1}}^{\beta_{1}} \ldots \lambda_{v_{j v}}^{\beta_{v}}$ for $1 \leq \mathrm{j}_{1} \leq \mathrm{n}_{1}, \ldots, 1 \leq \mathrm{j}_{\mathrm{v}} \leq \mathrm{n}_{\mathrm{v}}$

Corollary 3.8.([9]) The adjacency matrix $\mathrm{A}(\Sigma)$ of the Cartesian product $\Sigma=\Sigma_{1} \times \cdots \times \Sigma_{v}$ of $v$ signed graphs is $A_{1} \otimes I_{n 2} \otimes \cdots \otimes I_{n v}+I_{n 1} \otimes A_{2} \otimes \cdots \otimes I_{n v}+\cdots+I_{n 1} \otimes$ $\mathrm{I}_{\mathrm{n} 2} \otimes \cdots \otimes \mathrm{A}_{\mathrm{v}}$.

The eigenvalues of $\Sigma$ are the sums of the eigenvalues of the $\Sigma_{\mathrm{i}}$; i.e.,

$$
\lambda_{\mathrm{j} 1 \mathrm{j} 2 \ldots \mathrm{jv}}(\Sigma)=\lambda_{\mathrm{j} 1}\left(\Sigma_{1}\right)+\cdots+\lambda_{\mathrm{jv}}\left(\Sigma_{v}\right) .
$$

Proof. The corollary is immediate from Theorem 3.7 except the criterion for strict inequality, which is slightly stronger than that of Theorem 3.7 and is proved similarly.

\subsection{Balance Of The Cvetkovi'C' Product And The Cartesian Product}

The eigenvalues provide a short proof that the Cartesian product is balanced if and only if all constituents are balanced. Balance is important because, by Acharya's theorem, it causes the eigenvalues and energy (of both adjacency and Laplacian matrices) to be identical to those of the underlying unsigned graph, and therefore not interesting for themselves. We begin with a general theorem that provides a sufficient but not a necessary condition for balance of a Cvetkovi'c' product. 


\section{Available online at $w w w . i j r a t . o r g$}

Theorem 3.9.([9]) A Cvetkovi'c' product $\Sigma=\operatorname{NEPS}\left(\Sigma_{1}, \ldots, \Sigma_{v} ; \mathrm{B}\right)$ is balanced if $\Sigma_{1}, \ldots, \Sigma_{v}$ are all balanced.

Conversely, suppose B contains the vector $\beta_{i}=(0, \ldots, 0,1,0, \ldots, 0)$ with 1 in the ith position and 0 elsewhere. If $\Sigma_{\mathrm{i}}$ is unbalanced, $\Sigma$ is also unbalanced. The first part of Theorem 3.9 does not have a general converse. A counterexample is $\Sigma=$ $\operatorname{NEPS}\left(-\mathrm{G}_{1},+\mathrm{K}_{2} ; \mathrm{B}_{2}\right)$, where $\mathrm{B}_{2}=\{(1,1)\} . \Sigma$ is bipartite and all negative; therefore it is always balanced. However, $-\mathrm{G}_{1}$ is balanced only when $\mathrm{G}_{1}$ is bipartite. It is an open problem to determine which bases $\mathrm{B}$ have the property that for every Cvetkovi'c product $\Sigma=$ $\operatorname{NEPS}\left(\Sigma_{1}, \ldots, \Sigma_{v} ; \mathrm{B}\right)$ with basis $\mathrm{B}, \Sigma$ is balanced if and only if all the factors $\Sigma_{\mathrm{i}}$ are balanced. There is one important case in which there is such an if-and-only-if theorem.

Theorem 3.10. ([9])The following three statements about the Cartesian product $\Sigma=\Sigma_{1} \times \cdots \times \Sigma_{v}$ are equivalent.

(1) $\Sigma$ is balanced.

(2) All of $\Sigma_{1}, \ldots, \Sigma_{v} \quad$ are balanced.

(3) $\Sigma$ and its underlying graph $G$ have the same spectrum.

Corollary 3.11. Let $\Sigma=\Sigma_{1} \times \cdots \times \Sigma_{v}$. Then $\mathrm{b}(\Sigma)=\mathrm{b}\left(\Sigma_{1}\right) \cdots \mathrm{b}\left(\Sigma_{v}\right)$.

\subsection{Laplacian Matrix, Eigenvalues And Energy Of The Cartesian Product}

The formula for the Laplacian matrix of the Cartesian product is like that for the adjacency matrix. We write $\lambda^{\mathrm{L}}{ }_{\mathrm{i}}$ and $\mu^{\mathrm{L}}$ for the Laplacian eigenvalues of $\Sigma_{1}$ and $\Sigma_{2}$, respectively.

Theorem 3.12.([9]) The degree matrix of a Cvetkovi'c' product $\mathrm{G}=\operatorname{NEPS}\left(\mathrm{G}_{1}, \ldots, \mathrm{G}_{v} ; B\right)$ of graphs $\mathrm{G}_{\mathrm{i}}$ of order $\mathrm{n}_{\mathrm{i}}, 1 \leq \mathrm{i} \leq \mathrm{v}$, is

$$
\mathrm{D}(\mathrm{G})={ }^{\mathrm{X}} \mathrm{D}\left(\mathrm{G}_{1}\right)^{\beta 1} \otimes \cdots \otimes \mathrm{D}\left(\mathrm{G}_{v}\right)^{\beta v} .
$$

The average degree of the product is $\overline{\mathrm{d}}(\mathrm{G})=\sum_{\beta \in \mathrm{B}} \prod_{\mathrm{i}=1}^{\mathrm{v}} \overline{\mathrm{d}}\left(\mathrm{G}_{\mathrm{i}}\right)^{\beta_{\mathrm{i}}}$.

In particular, the degree matrix of the Cartesian product is

$\mathrm{D}\left(\mathrm{G}_{1} \times \ldots \times \mathrm{G}_{\mathrm{v}}\right)=\mathrm{D}(\mathrm{G} 1) \otimes \mathrm{I}_{\mathrm{n} 2} \ldots \ldots \otimes \mathrm{I}_{\mathrm{nv}}+\mathrm{I}_{\mathrm{n} 1}$

$\otimes D\left(G_{2}\right) \otimes \ldots . \otimes I_{n v}+\ldots .+I_{n 1} \otimes I n 2 \otimes \ldots \ldots \otimes$

$\mathrm{D}\left(\mathrm{G}_{\mathrm{v}}\right)$.

The average degree is $\overline{\mathrm{d}}(\mathrm{G})=\sum_{\mathrm{i}=1}^{\mathrm{v}} \overline{\mathrm{d}}\left(\mathrm{G}_{\mathrm{i}}\right)$.

Theorem 3.13.([9]) Given signed graphs $\Sigma_{1}$ of order $\mathrm{n}_{1}$, $\ldots, \Sigma_{v}$ of order $\mathrm{n}_{v}$, the Laplacian matrix of the Cartesian product $\Sigma=\Sigma_{1} \times \cdots \times \Sigma_{v}$ is

$\mathrm{L}(\Sigma)=\mathrm{L}\left(\Sigma_{1}\right) \otimes \mathrm{I}_{\mathrm{n} 2} \otimes \cdots \otimes \mathrm{I}_{\mathrm{n} v}+\cdots+\mathrm{I}_{\mathrm{n} 1} \otimes \mathrm{I}_{\mathrm{n} 2} \otimes \cdots \otimes$ $\mathrm{L}\left(\Sigma_{v}\right)$.

The Laplacian eigenvalues of the Cartesian product are the sums of those of all the factors $\Sigma_{\mathrm{i}}$; i.e., $\lambda_{\mathrm{jk}}^{\mathrm{L}}(\Sigma)=\sum_{\mathrm{i}=1}^{\mathrm{v}} \lambda_{\mathrm{ij}}^{\mathrm{L}}$.

Theorem 3.13 does not generalize to other Cvetkovi'c' products. For a vector $\beta$ of weight $\sum_{i=1}^{v} \beta_{i}>1$, the Laplacian of $\Sigma_{\beta}=\operatorname{NEPS}\left(\Sigma_{1}, \ldots, \Sigma_{v} ; \beta\right)$ is

$$
\begin{aligned}
\mathrm{D}\left(\Sigma_{\beta}\right)-\mathrm{A}\left(\Sigma_{\beta}\right) & =\mathrm{D}\left(\Sigma_{1}\right)^{\beta_{1}} \otimes \ldots \otimes \mathrm{D}\left(\Sigma_{\mathrm{v}}\right)^{\beta_{\mathrm{v}}} \\
& -\mathrm{A}\left(\Sigma_{1}\right)^{\beta_{1}} \otimes \ldots \otimes \mathrm{A}\left(\Sigma_{\mathrm{v}}\right)^{\beta_{\mathrm{v}}}
\end{aligned}
$$

will not combine by linear combination into a product of Laplacian matrices. The general product $\operatorname{NEPS}\left(\Sigma_{1}, \ldots, \Sigma_{v} ; \mathrm{B}\right)$ where B contains a vector of weight $>1$ has the same difficulty. The only Cvetkovi'c' product in which no vector has weight $>1$ is the Cartesian product.

\section{SCOPE FOR FURTHER EXTENSION TO SKEW-GAIN GRAPHS}

Many of the concepts and results described in the previous sections can be naturally extended to the realm of skew-gain graphs which are defined below. Before that we need the definition of anti-involution. We call a function $\mathrm{f}: \Gamma \rightarrow \Gamma$ to be an involution if $f(f(x))=x$ for all $x \in \Gamma$. The collection of all antiinvolutions is denoted by $\operatorname{Inv}(\Gamma)$.

Definition 4.1. Let $G=(V, \vec{E})$ be a graph with some prescribed orientation and $\Gamma$ be an arbitrary group. If $\mathrm{f}$ $\in \operatorname{Inv}(\Gamma)$ then the skew-gain graph $\Phi_{\mathrm{f}}=(\mathrm{G}, \Gamma, \varphi, \mathrm{f})$ is such that the skew gain function $\varphi: \overrightarrow{\mathrm{E}} \rightarrow \Gamma$ satisfies $\varphi$ $(\overrightarrow{v u})=f(\varphi(\overrightarrow{u v}))$.

Note that skew-gain graphs encompass all the structures like graphs, signed graphs and gain graphs by choosing proper anti-involutions for each case.

\section{REFERENCES}

[1] B. D. Acharya, Spectral criterion for cycle balance in networks. J. Graph Theory 4 (1980) 1-11.

[2] R. Balakrishnan, The energy of a graph. Linear Algebra Appl. 387 (2004) 287-295.

[3] Francisco Barahona, On the computational complexity of Ising spin glass models. J. Phys. A: Math. Gen. 15 (1982) 3241-3253.

[4] D. Cartwright and F. Harary, Structural balance: A generalization of Heider's theory. Psychological Rev. 63 (1956) 277-293.

[5] Grafovi i njihovi spektri. [Graphs and their Spectra.] Univ. Beograd Publ. Elektrotehn. Fak., Ser. Mat. Fiz., No. 354-356 (1970), 150.

[6] Drago`s M. Cvetkovi'c, Michael Doob, and Horst Sachs, Spectra of Graphs: Theory and Application. VEB Deutscher Verlag der Wissenschaften, Berlin, and Academic Press, New York, 1980.

[7] D. Cvetkovi'c,' P. Rowlinson, and S. K. Simi'c, Signless Laplacians of finite graphs. Linear Algebra Appl. 423 (2007) 155-171.

[8] K. A. Germina and Shahul Hameed K, On signed paths, signed cycles and their energies. Applied Mathematical Sciences, 4 (70) (2010) 3455 - 3466.

[9] K. A. Germina, Shahul Hameed K and Thomas Zaslavsky, On product and line graphs of signed graphs, their eigenvalues and 


\section{Available online at www.ijrat.org}

energy. Linear Algebra and its Appl., 435 (2011) 2432-2450.

[10] Shahul Hameed K and K. A. Germina, Balance in gain graphs. A spectral analysis. Linear Algebra and its Appl., 436 (2012) 1114-1121.

[11] Shahul Hameed K and K. A. Germina, On composition of signed graphs. Discussiones Mathematicae Graph Theory, 32 (2012) 507516.

[12] Shahul Hameed K and K. A. Germina, Balance in certain gain graph products. Journal of Combinatorics, Information and System Sciences, 37 (2012) 205 - 216.

[13] Shahul Hameed K, Viji Paul and K. A. Germina, On coregular signed graphs. The Australasian Journal of Combinatorics, 62(1) (2015) 8-17.

[14] M. K. Gill and B. D. Acharya, A recurrence formula for computing the characteristic polynomial of a sigraph. J. Combin. Inform. Syst. Sci. 5 (1980) 68-72.

[15] I. Gutman, The energy of a graph. Ber. Math.Stat. Sekt. Forschungszent. Graz 103 (1978) $1-22$.

[16]I. Gutman, The energy of a graph: old and new results. In: Algebraic Combinatorics and Applications (Go“ssweinstein,1999). Springer, Berlin, 2001, pp. 196-211.

[17] I. Gutman and B. Zhou, Laplacian energy of a graph. Linear Algebra Appl. 414 (2006) 29-37.
[18]I. Gutman and O. E. Polansky, Mathematical Concepts in Organic Chemistry. Springer-Verlag, Berlin, 1986, pp. 54-55.

[19]F. Harary, Graph Theory. Addison Wesley, Reading, Mass., 1972.

[20]E. Heilbronner, Hu“ckel molecular orbitals of Mo"bius-type conformations of annulenes. Tetrahedron Lett. 5 (1964) 1923-1928.

[21] A. M. Mathai, On adjacency matrices of simple signed cyclic connected graphs. Submitted, 2010.

[22] Ramachandra Rao A and P. Bhimasankaram, Linear Algebra, Second ed., Hindustan Book Agency, 2000.

[23]E. Sampathkumar, Graph Structures. DST Annual Progress Report \# SR/S4/MS:235/02, 2005.

[24]A. J. Schwenk and R. J. Wilson, On the eigenvalues of graphs. In: Lowell W. Beineke and Robin J. Wilson, eds., Selected Topics in Graph Theory, Academic Press, London, 1978, Ch. 11, pp. 307-336.

[25] G. Sabidussi, The composition of graphs. Duke Math Journal. Vol.26(1959),693-696.

[26]G. Sabidussi, The lexicographic product of graphs. Duke Math Journal. Vol.28(1961),573-578.

[27] T. Zaslavsky, Signed graphs. Discrete Appl. Math. 4 (1982) 47-74. Erratum. Discrete Appl. Math. 5 (1983) 248. 